\title{
Cardiac magnetic resonance imaging-based myocardial strain study for evaluation of cardiotoxicity in breast cancer patients treated with trastuzumab: A pilot study to evaluate the feasibility of the method
}

Shintaro Nakano ${ }^{1}$, Masahiro Takahashi², Fumiko Kimura ${ }^{2}$, Taiki Senoo ${ }^{2}$, Toshiaki Saeki ${ }^{3}$, Shigeto Ueda ${ }^{3}$, Jun Tanno ${ }^{1}$, Takaaki Senbonmatsu ${ }^{1}$, Takatoshi Kasai ${ }^{4}$, Shigeyuki Nishimura ${ }^{1}$

${ }^{1}$ Department of Cardiology, International Medical Center, Saitama Medical University, Saitama, Japan

${ }^{2}$ Department of Diagnostic Radiology, International Medical Center,

Saitama Medical University, Saitama, Japan

${ }^{3}$ Department of Breast Oncology, International Medical Center,

Saitama Medical University, Saitama, Japan

${ }^{4}$ Cardiovascular Respiratory Sleep Medicine, Department of Cardiovascular Medicine,

Juntendo University Graduate School of Medicine, Tokyo, Japan

\begin{abstract}
Background: Trastuzumab, used to treat breast cancer overexpressing human epidermal growth factor receptor 2, may be cardiotoxic. Cardiac magnetic resonance (CMR) imaging with myocardial strain studies has been used to evaluate subclinical biventricular myocardial changes, however, its clinical utility during chemotherapy has not been evaluated.

Methods: The clinical outcomes, CMR and cardiac biomarkers of 9 women aged $62.3 \pm 12.6$ years with early or locally advanced breast cancer were evaluated at baseline, and at 3, 6 and 12 months after the initiation of trastuzumab.
\end{abstract}

Results: None of the patients developed heart failure or elevated serum cardiac biomarkers. Global left ventricular $(L V)$ peak systolic longitudinal and circumferential strains were significantly decreased at 6 months (longitudinal strains, $-21.1 \pm 1.7 \%$ [baseline] vs. $-19.5 \pm 1.0 \%$ [6 months], $p=0.039$, and circumferential strains, $-23.4 \pm 1.8 \%$ [baseline] vs. $-21.6 \pm 2.5 \%$ [6 months], $p=0.036$ ). These changes were analogous to those observed in the LV ejection fraction. Right ventricular $(R V)$ free wall peak systolic circumferential strains were decreased at 6 months $(-20.9 \% \pm 2.4 \%$ [baseline] vs. $-19.1 \% \pm 2.3 \%$ [6 months], $p=0.049)$, whereas $R V$ longitudinal strains and ejection fraction remained unchanged. The LV longitudinal strain was the most reproducible of the 4 peak strain parameters.

Conclusions: The LV longitudinal and circumferential strains measured by CMR decreased during trastuzumab therapy, although their predictive value for later heart failure or association with RV parameters was not determined. These techniques may be a useful means of diagnosing and monitoring trastuzumab-related cardiotoxicity. (Cardiol J 2016; 23, 3: 270-280)

Key words: cardiac magnetic resonance, myocardial strain, trastuzumab, breast cancer, cardiotoxicity

Address for correspondence: Shintaro Nakano, MD, Department of Cardiology, International Medical Center, Saitama Medical University 1397-1 Yamane, Hidaka, Saitama 350-1298, Japan, tel: +81-42-984-4111, fax: +81-42-984-4591, e-mail: snakano@saitama-med.ac.jp

Received: 24.12.2015 Accepted: 07.05.2016 


\section{Introduction}

Trastuzumab, a humanized monoclonal antibody against the extracellular domain of human epidermal growth factor receptor 2 (HER2), improves clinical outcomes in patients with breast cancer overexpressing HER2. Adjuvant therapy using trastuzumab reportedly reduces the risk of relapse by about a half and the risk of death by a third; a substantial body of evidence for its benefits has led to trastuzumab becoming a first line treatment for early-stage HER2-positive breast cancer [1,2].

Although generally well tolerated, trastuzumab is cardiotoxic in $2-27 \%$ of patients; the incidence varies according to the definition used and concomitant chemotherapy $[1,3]$. The Cardiac Review and Evaluation Committee (CREC) that examined trastuzumab-associated cardiotoxicity defined cardiotoxicity as a $\geq 5 \%$ reduction in left ventricular (LV) ejection fraction (LVEF) to $<55 \%$ in symptomatic patients, or a $\geq 10 \%$ reduction in $\mathrm{LVEF}$ to $<55 \%$ in asymptomatic patients [4]. Nevertheless, LVEF measured by echocardiography may not be sufficiently specific or sensitive to detect cardiotoxicity reliably $[5,6]$. Deformation parameters such as myocardial strain have been used to detect subclinical myocardial dysfunction before reduced LVEF is identifiable on echocardiography in patients with breast cancer receiving trastuzumab [6-9].

Cardiac magnetic resonance (CMR) imaging provides excellent images of the heart, allowing 3-dimensional assessments of LVEF and ventricular volumetric function [10]. Although its high cost and lack of availability precludes its routine clinical use, CMR has been validated for serial monitoring of LVEF and volume in patients with breast cancer receiving trastuzumab, as well as for the characterization of myocardial tissue changes $[7,11,12]$. Recently, CMR has been also used to monitor right ventricular (RV) ejection fraction (RVEF) in cardiotoxicity [13]. Additionally, CMR can now be used to measure deformation parameters in conjugation with strain-encoded imaging (SENC) [14], in which objective color-coded biventricular longitudinal and circumferential myocardial strain patterns are obtained in several heartbeats and breath-holds.

We hypothesized that CMR-based measurement of myocardial strains, in addition to the conventional biventricular CMR parameters, may be a reliable means of assessing change in cardiac function during chemotherapy. Our pilot study aimed to establish whether CMR with myocardial strain studies might have clinical utility in patients with breast cancer treated with trastuzumab.

\section{Methods}

\section{Patient selection and study design}

Of patients newly diagnosed with HER2-overexpressing breast cancer between April 2012 and October 2014 in our institute, 16 women consented to participate in our pilot study. The exclusion criteria were: 1 ) artificial pacemaker implantation, 2) chronic arrhythmia such as atrial fibrillation, 3) chronic kidney disease (estimated glomerular filtration rate $<30 \mathrm{~mL} / \mathrm{min} / 1.73 \mathrm{~m}^{2}$ ), and 4) metastatic cancer. Intravenous trastuzumab was administered at a loading dose of $8 \mathrm{mg} / \mathrm{kg}$ followed by $6 \mathrm{mg} / \mathrm{kg}$ every 3 weeks (a total of 18 doses) in all patients. Physical examination, echocardiography, CMR and blood tests were performed before the initiation of trastuzumab, and 3 months ( \pm 6 weeks), 6 months ( \pm 6 weeks) and 12 months ( \pm 8 weeks) afterwards. Cardiac function was evaluated by CMR at each of the 4 time points, and the relationships with echocardiographic parameters, biomarkers and clinical indices were evaluated.

\section{Data collected at baseline}

Patients' demographic characteristics, including age and body mass index, were recorded at baseline. The cardiovascular history was taken and risk factors, including hypertension, dyslipidemia, diabetes mellitus, chronic atrial fibrillation, chronic kidney disease, coronary artery disease (myocardial infarction or angina pectoris) and current smoking were assessed (see Supplementary Materials). Cancer-related variables, including side of the breast cancer, stage (early or locally advanced), surgical treatment, radiotherapy, and other concomitant chemotherapy were recorded. Vital signs, including systolic and diastolic blood pressure, and heart rate at rest were recorded. Echocardiographic variables, including LV enddiastolic dimension, end-systolic dimension, and intra-ventricular septum and LV posterior wall thickness were measured, and the presence of significant valvular disease was evaluated. LVEF was calculated using the Teichholz and modified Simpson's methods $[15,16]$.

\section{Cardiac magnetic resonance imaging}

Cardiac magnetic resonance imaging was performed within 28 days of echocardiography using a 3.0 Tesla MR imaging scanner (Archieva 


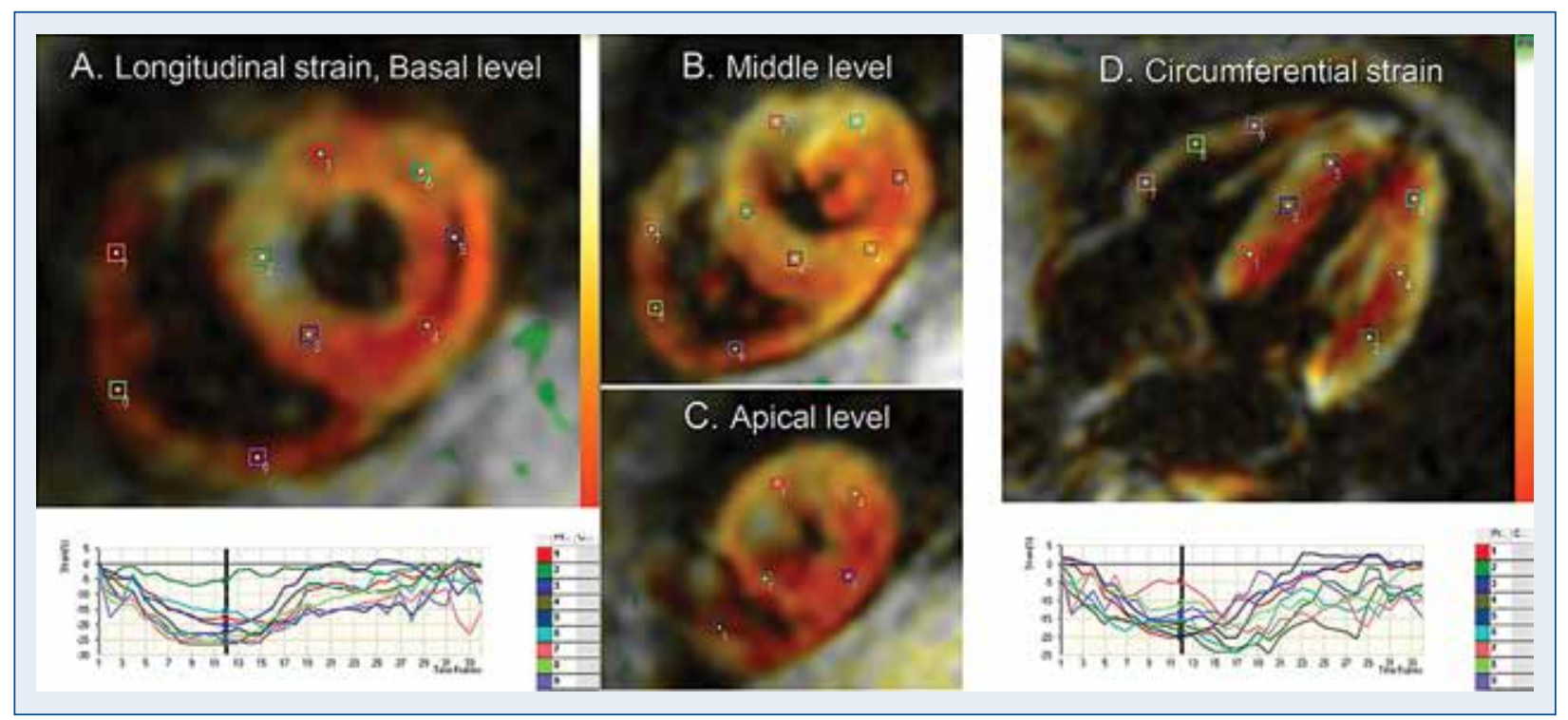

Figure 1. Measurement of peak systolic strain. Myocardial strain was measured using strain-encoded cardiac magnetic resonance imaging (SENC-CMR). The short-axis view shows longitudinal myocardial strain and the 4-chamber view shows circumferential strain. Areas of myocardium with severely impaired or absent strain are shown in white, and areas of myocardium with normal strain are shown in red. Global strain was defined as the mean of peak systolic strain of all allocated segments; A-C. Longitudinal strain, in the basal, middle and apical levels; D. Circumferential strain.

3.0T TX, Philips Medical Systems, Best, The Netherlands) with a 32-element phased-array cardiac coil. Cine studies using steady-state free precession sequence in the LV vertical long-axis, 4-chamber view, and sequential short-axis slices from the mitral valve to the apical levels were obtained at 4 time points (Supplementary Table S1). T2-weighted short tau inversion recovery (STIR) and late-gadolinium enhancement (LGE) images after intravenous administration of $0.15 \mathrm{mmol} / \mathrm{kg}$ meglumine gadoterate (Magnescope; Guerbet Japan, Tokyo, Japan) were obtained at baseline only, to exclude pre-existing cardiac disease or irreversible myocardial injury that had developed after previous chemotherapy. LV end-diastolic volume index (LVEDVI in $\mathrm{mL} / \mathrm{m}^{2}$ indexed by body surface area), LV end-systolic volume index (LVESVI in $\mathrm{mL} / \mathrm{m}^{2}$ ) and $\mathrm{LV}$ mass index (LVMI in $\mathrm{g} / \mathrm{m}^{2}$ ) were measured as previously described [17] using a Philips Extended MR Workspace 2.6.3.4 (Philips Medical Systems). Briefly, using the stack of short-axis cine images, the most basal and apical slices of the LV were determined, and LV endocardial and epicardial borders were manually traced to obtain LVEDVI, LVESVI and LVMI. LVEF was calculated using the blood volume method, which excluded portions of papillary and trabecular muscle. Using the same images, RV end-diastolic volume index (RVEDVI in $\mathrm{mL} / \mathrm{m}^{2}$ ) and
$\mathrm{RV}$ end-systolic volume index (RVESVI in $\mathrm{mL} / \mathrm{m}^{2}$ ) were measured [18]; RVEF was calculated using the blood volume method.

Myocardial strains were evaluated using SENCCMR (version 3.0; Diagnosoft, Palo Alto, CA, Fig. 1), based on the acquisition of two images of differing frequency modulation; superposition of low- and high-tuning images allowed a SENC strain map to be plotted, which was overlaid pixel-by-pixel on the anatomical images $[14,19]$. In contrast with conventional tagging, in SENC-CMR the gradient is applied in the slice selection direction [14], therefore, the short-axis view shows longitudinal myocardial strain and the 4-chamber view shows circumferential strain. Areas of myocardium with severely impaired or absent strain are shown in white, and areas of myocardium with normal strain are shown in red. LV longitudinal strain was measured at the basal, middle, and apical levels of the short-axis view; a region of interest was plotted in the myocardial middle-layer of each myocardial segment and the minimum value during systole (i.e., peak systolic strain) was recorded. Using the same images, RV longitudinal and circumferential strains were measured in regions of interest plotted in the $\mathrm{RV}$ free wall using a method modified from a previous report [20]. Global strain was defined as the mean peak systolic strain of all allocated segments (16 segments for LV longitudinal strain, 6 for circumferential strain, 
7 segments for RV free wall longitudinal strain, and 3 for circumferential strain). Left ventricular wall motion at baseline was evaluated by the agreement of two observers using a 17-segment model [21]. The detailed CMR protocol is described in Supplementary Table S1.

\section{Other data collected}

Clinical signs and symptoms of heart failure (such as dyspnea and peripheral edema), and the serum concentrations of troponin-I (Architect STAT Troponin-I assay, Abbott Diagnostics, Illinois, USA), ventricular myosin light chain (SRL, Tokyo, Japan), and plasma B-type natriuretic peptide (BNP) (TOSOH, Osaka, Japan) were measured at the same intervals (see Supplementary Materials). Values below the lower limit of detection were treated as zero. LVEF was also measured by echocardiography at each time point.

\section{Statistical analysis}

Continuous data are expressed as the mean \pm standard deviation (SD) and categorical data as the number (proportion). Independent continuous variables at the 3 different time points (3, 6 and 12 months) were compared with the baseline data using the paired t-test. Intra- and inter-observer variability was evaluated for reproducibility using Pearson's correlation analysis; standardized dispersion was expressed as coefficient of variation. Inter-modality comparison between CMR and echocardiography was also evaluated using Pearson's correlation analysis. A p value $<0.05$ was considered statistically significant. All analyses were performed using JMP V.10.0.0 (SAS Institute Inc., Cary, NC).

\section{Ethics approval}

The study was conducted according to the Declaration of Helsinki and approved by the Institutional Review Board of the International Medical Centre of Saitama Medical University, Japan (reference number 11-067).

\section{Results}

Baseline characteristics, and changes in echocardiographic findings and cardiac biomarkers from baseline

Of the 16 women enrolled, 7 were excluded as they did not undergo CMR at each of the 4 time points due to: progression of cancer $(n=2)$; interruption by surgery $(\mathrm{n}=3)$, and withdrawal of consent to participate $(n=2)$. The data of remaining 9 patients were analyzed. The mean age of the patients was $62.3 \pm 12.6$ years (Table 1 ). Two had essential hypertension (1 was treated with an angiotensin converting enzyme inhibitor), 1 had diabetes mellitus and 1 had dyslipidemia, but none had previous or co-existing heart failure. Eight $(88.9 \%)$ patients had a right-sided tumor, and $8(88.9 \%)$ underwent either partial or total mastectomy. Intravenous epirubicin was administered prior to trastuzumab in $6(66.7 \%)$ patients, with a cumulative dose of $360 \mathrm{mg} / \mathrm{m}^{2}$ in 5 patients and $342 \mathrm{mg} / \mathrm{m}^{2}$ in 1 patient (in whom the dose was reduced owing to inflammation of the throat). Echocardiography at baseline demonstrated preserved $\operatorname{LVEF}(71.8 \% \pm 7.4 \%$, range: $62.7-86.2 \%)$ and the absence of significant valvular disease in all patients. The serum concentrations of troponin I, myosin light chain, and plasma BNP were not elevated at baseline.

Throughout the observation period, none of the patients developed symptoms of heart failure, nor did any exhibit an LVEF $<55 \%$ measured by echocardiography (Supplementary Table S2). In all patients, the serum concentrations of troponin I and myosin light chain remained within their normal ranges, and the plasma concentration of BNP remained lower than $\leq 100 \mathrm{pg} / \mathrm{mL}$, the validated cutoff value for suspected acute heart failure [22]. In 1 patient, borderline LVEF reduction was treated with an oral $\beta$-blocker, but this was discontinued almost immediately because of faintness.

\section{Change in CMR parameters}

There was no evidence of myocardial LGE or high signal intensity in T2-weighted short tau inversion recovery images, or abnormal LV motion at baseline in any of the participants. The mean LVESVI increased 3 months after the initiation of trastuzumab and remained high until 12 months. The LVEF (68.4 $\pm 6.6 \%$, range $57.3-80.8 \%$ at baseline) significantly decreased by 6 months $(61.7 \pm$ $\pm 8.7 \%, \mathrm{p}=0.004$ compared with baseline), and remained low at 12 months $(62.9 \pm 7.8 \%, \mathrm{p}=0.002$ compared with baseline). Over the 12-month study period, minimum LVEF measured by CMR fell to $54.3 \%$ and $53.9 \%$ in 2 cases, but the corresponding LVEF measured by echocardiography was $61.9 \%$ and $55.9 \%$, respectively. Other ventricular parameters such as the LVEDVI and LVMI remained unchanged throughout the observation period. The conventional RV parameters, such as RVEDVI, RVESVI, and RVEF remained unchanged (Fig. 2).

Global LV peak systolic longitudinal and circumferential strains significantly decreased by 6 months (longitudinal strain, $-21.1 \pm 1.7 \%$ at 
Table 1. Baseline characteristics (total: $n=9$ ).

\begin{tabular}{|c|c|}
\hline \multicolumn{2}{|l|}{ Demographics } \\
\hline Age [years] & $62.3 \pm 12.6$ \\
\hline Body mass index $\left[\mathrm{kg} / \mathrm{m}^{2}\right]$ & $22.1 \pm 3.4$ \\
\hline \multicolumn{2}{|l|}{ Cardiovascular history and risk factors } \\
\hline Hypertension & $2(22.2 \%)$ \\
\hline Diabetes mellitus & $1(11.1 \%)$ \\
\hline Dyslipidemia & $1(11.1 \%)$ \\
\hline Chronic atrial fibrillation & $0(0.0 \%)$ \\
\hline Chronic kidney disease & $0(0.0 \%)$ \\
\hline Coronary artery disease & $0(0.0 \%)$ \\
\hline Current smoking & $0(0.0 \%)$ \\
\hline \multicolumn{2}{|l|}{ Cancer-related variables } \\
\hline \multicolumn{2}{|l|}{ Breast cancer side: } \\
\hline Right & $8(88.9 \%)$ \\
\hline Bilateral & $0(0.0 \%)$ \\
\hline Stage: & $0(0.0 \%)$ \\
\hline Early & $3(37.5 \%)$ \\
\hline Locally advanced & $5(62.5 \%)$ \\
\hline Metastatic & $0(0.0 \%)$ \\
\hline \multicolumn{2}{|l|}{ Surgery: } \\
\hline Partial mastectomy $+\mathrm{Sn}$ & $3(33.3 \%)$ \\
\hline Partial mastectomy $+A x$ & $0(0.0 \%)$ \\
\hline Total mastectomy $+\mathrm{Sn}$ & $2(22.2 \%)$ \\
\hline Total mastectomy $+\mathrm{Ax}$ & $3(33.3 \%)$ \\
\hline Radiotherapy & $5(55.6 \%)$ \\
\hline \multicolumn{2}{|l|}{ Other chemotherapy: } \\
\hline Epirubicin & $6(66.7 \%)$ \\
\hline Docetaxel & $3(33.3 \%)$ \\
\hline Cyclophosphamide & $8(88.9 \%)$ \\
\hline Carboplatin & $1(11.1 \%)$ \\
\hline \multicolumn{2}{|l|}{ Vital signs at baseline } \\
\hline Systolic BP [mm Hg] & $125.6 \pm 9.2$ \\
\hline Diastolic BP [mm Hg] & $79.4 \pm 9.2$ \\
\hline Heart rate $[\mathrm{bpm}]$ & $77.8 \pm 7.9$ \\
\hline \multicolumn{2}{|l|}{ Echocardiographic parameters at baseline } \\
\hline LV end-diastolic dimension [mm] & $43.2 \pm 2.9$ \\
\hline LV end-systolic dimension [mm] & $25.3 \pm 2.7$ \\
\hline LV ejection fraction [\%] & $71.8 \pm 7.4$ \\
\hline Intra-ventricular septum thickness [mm] & $9.5 \pm 1.1$ \\
\hline LV posterior wall thickness [mm] & $9.3 \pm 0.9$ \\
\hline Significant valvular disease & $0(0.0 \%)$ \\
\hline \multicolumn{2}{|l|}{ Cardiac biomarkers at baseline } \\
\hline Troponin-I [ng/mL] & $0.02 \pm 0.00$ \\
\hline Myosin light chain [ng/mL] & $1.33 \pm 0.41$ \\
\hline B-type natriuretic peptide $[\mathrm{pg} / \mathrm{mL}]$ & $23.1 \pm 13.5$ \\
\hline
\end{tabular}

Continuous variables are described as the mean \pm standard deviation and categorical variables as number (proportion); $A x$ - axillary clearance; BP - blood pressure; LV - left ventricular; $\mathrm{Sn}$ - sentinel lymph node biopsy baseline vs. $-19.5 \pm 1.0 \%$ at 6 months, $\mathrm{p}=0.039$; circumferential strains, $-23.4 \pm 1.8 \%$ at baseline vs. $-21.6 \pm 2.5 \%$ at 6 months, $\mathrm{p}=0.036)$. RV free wall peak systolic circumferential strains had decreased by 6 months $(-20.9 \pm 2.4 \%$ at baseline vs. $-19.1 \pm 2.3 \%$ at 6 months, $\mathrm{p}=0.049)$, while longitudinal strain remained unchanged (Fig. 2; Supplementary Table S3). Images demonstrating a typical reduction in LV longitudinal strain are shown in Figure 3.

The intra-observer (measured by Dr. Nakano) and inter-observer (measured by Drs. Nakano and Tanno) correlation coefficients, and the coefficient of variation are shown in Table 2 . The correlation coefficients between the LVEF measured by CMR and that by echocardiography using the modified Simpson method (26 of 36 studies), and CMR and echocardiography using the Teichholz method were $0.58(\mathrm{p}=0.004)$ and $0.46(\mathrm{p}=0.004)$, respectively (Supplementary Fig. S1).

\section{Discussion}

Although the number of patients was limited, our pilot study is the first to have used CMR-based myocardial strain studies to undertake serial evaluations of cardiac function in a cohort of patients with breast cancer treated with trastuzumab.

We found that global LV peak systolic longitudinal and circumferential strains had significantly decreased by 6 months before recovering by 12 months. These changes were analogous to those of LVEF measured by CMR. LV longitudinal strain was most reproducible among the 4 peak strain parameters. We also found that RV free wall longitudinal strain remained unchanged, whereas circumferential strain decreased 6 months after the initiation of trastuzumab. The RV circumferential strain was, however, the least reproducible and the coefficient of variation was the greatest of the 4 ventricular peak systolic strain parameters.

Role of conventional CMR in the assessment of chemotherapy-related cardiotoxicity

Cardiac magnetic resonance imaging has recently been used to characterize myocardial tissue by LGE for patients with breast cancer being treated with chemotherapy $[7,23]$, as the presence of LGE may reflect irreversible myocardial injury [24]. In our study, no patient showed LGE at baseline, suggesting that no advanced myocardial damage had been induced by prior exposure to epirubicin. The mechanism of trastuzumab-related cardiotoxicity is unclear; it may be attributable to 


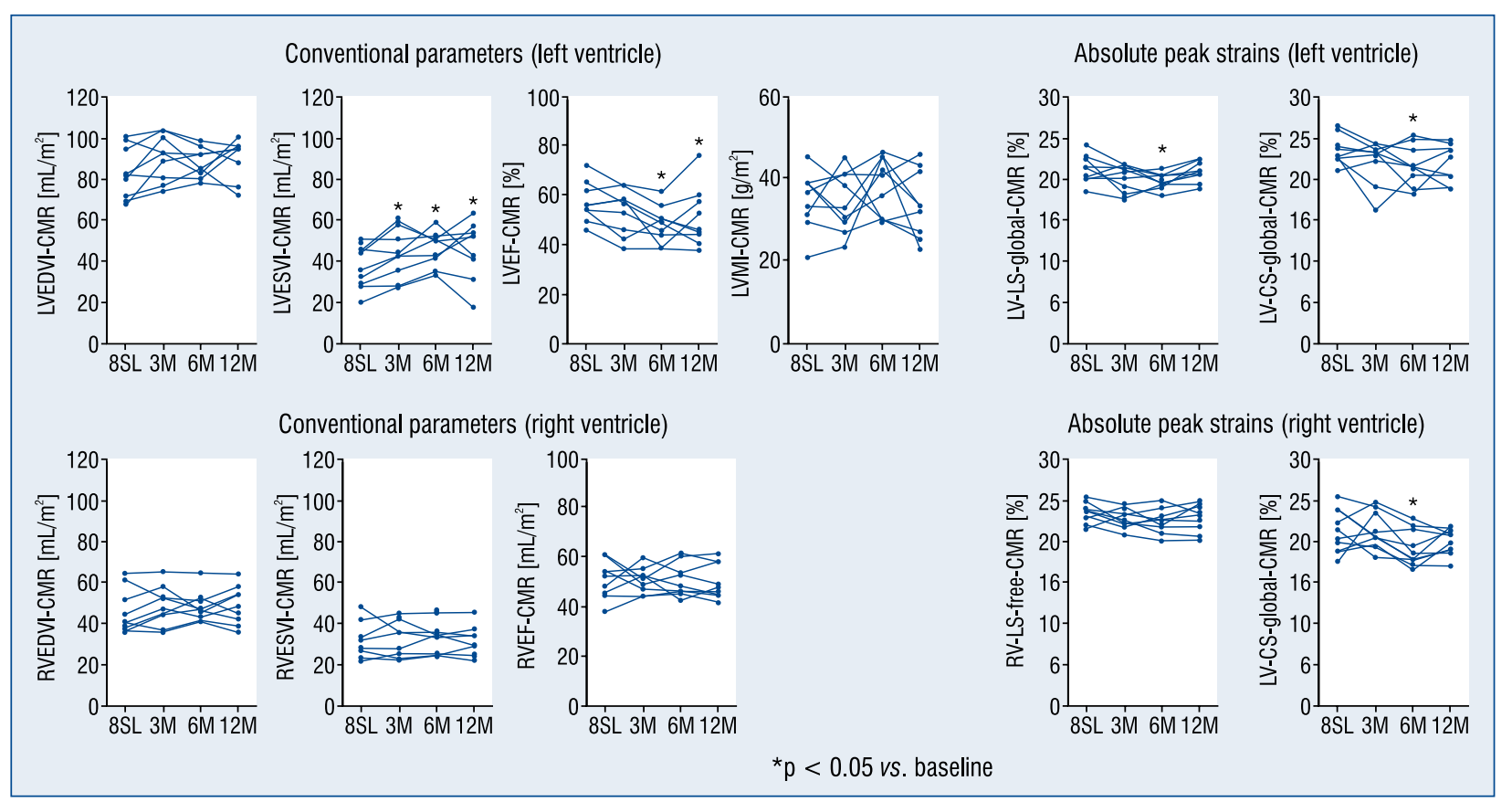

Figure 2. Cardiac magnetic resonance (CMR) imaging parameters. Variables at the three different time points $(3,6$ and 12 months) were compared with the baseline data using the paired t-test. Peak systolic myocardial strains are expressed as absolute values; ${ }^{*} p<0.05$ vs. baseline; LVEDVI — left ventricular end-diastolic volume index; LVESVI — left ventricular end-systolic volume index; LVMI — left ventricular mass index; LV-LS — left ventricular longitudinal strain; LV-CS — left ventricular circumferential strain; LVEF — left ventricular ejection fraction; RVEF — right ventricular ejection fraction; RVEDVI — right ventricular end-diastolic volume index; RVESVI — right ventricular end-systolic volume index; RV-LS — right ventricular longitudinal strain; RV-CS — right ventricular circumferential strain; $\mathrm{BSL}$ - baseline; $\mathrm{M}$ - months.

blockade of the HER2 in cardiomyocytes, representing largely reversible type II cardiotoxicity without ultrastructural abnormalities [2, 25]. None of the participants in our study exhibited progressive cardiac dysfunction. Still, the technique may be a useful means of evaluating irreversible myocardial injury in patients receiving trastuzumab following anthracycline, as prior anthracycline exposure is reportedly a risk factor for trastuzumab-related cardiotoxicity alongside concomitant radiation therapy and general cardiovascular risk factors (such as older age, obesity, smoking, dyslipidemia, hypertension, diabetes mellitus and history of coronary artery disease) $[3,26,27]$.

In our cohort of patients with breast cancer, the LVEF measured by CMR tended to decrease at 3 months, and had significantly decreased by 6 months after the initiation of trastuzumab. This finding is consistent with previous studies, which found the reduction in LVEF was first detectable at approximately 3 months and reached its peak between 6 and 9 months after the initiation of trastuzumab [7]. Although there is presently a limited role for the routine use of CMR in the evaluation of trastuzumab-related cardiotoxicity, it has several advantages over echocardiography, such as higher reproducibility and sensitivity [28]. Indeed, a substantial proportion of our echocardiographic studies ( 10 of $36,27.8 \%$ ) were of insufficient visual quality for data acquisition using the modified Simpson's method; therefore, only the Teichholz method was available in all studies. Moreover, the ability to obtain high-quality, 3-dimensional images with CMR may be particularly advantageous with breast cancer patients with left-sided disease, because the mass effect of cancer or scar tissue wound may further obscure echocardiographic views or a painful surgical wound may interfere with the proper placement of the ultrasound probe. Our study demonstrated a modest intra-modality discrepancy between LVEF measured by echocardiography and that by CMR, which was consistent with previous reports $[12,29,30]$. This inherent intra-modality discrepancy may be important in patients with borderline cardiotoxicity. In our study, neither of the 2 patients with minimum LVEF $<55 \%$ measured by CMR ( $54.3 \%$ and $53.9 \%$ each) would have met the CREC criteria for cardiotoxicity if only echocar- 


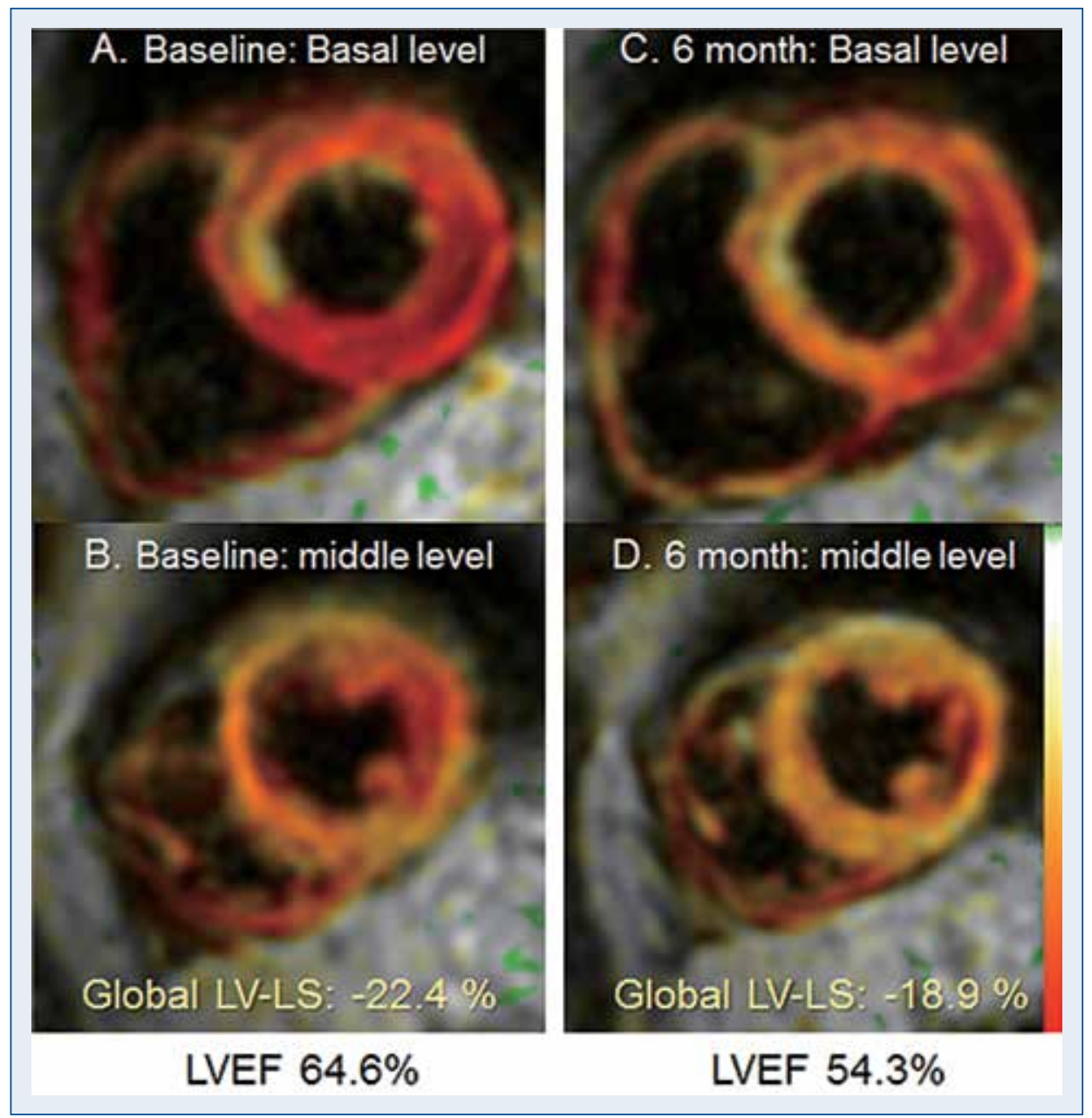

Figure 3. Representative changes in left ventricular longitudinal strain (LV-LS). A 60-year-old woman previously treated with epirubicin demonstrated reduction in global LV-LS at 6 months $(-22.4 \%$ to $-18.9 \%)$ concomitant with a decrease in left ventricular ejection fraction (LVEF). A. Baseline: basal level; B. Baseline: middle level; C. Six month: basal level; D. Six month: middle level.

Table 2. Reproducibility of peak systolic myocardial strain measured by strain-encoded cardiac magnetic resonance imaging.

\begin{tabular}{lccc}
\hline & Intra-observer variability & Inter-observer variability & Coefficient of variation \\
\hline LV, longitudinal & 0.968 & 0.939 & $-7.20 \%$ \\
LV, circumferential & 0.926 & 0.871 & $-10.34 \%$ \\
RV, longitudinal & 0.931 & 0.797 & $-5.94 \%$ \\
RV, circumferential & 0.905 & 0.757 & $-12.17 \%$ \\
\hline
\end{tabular}

Intra- and inter-observer variability was evaluated using Pearson's correlation; LV — left ventricle; RV — right ventricle

diography had been used to measure LVEF $(61.9 \%$ and $55.9 \%$, respectively).

Role of myocardial strain in the assessment of chemotherapy-related cardiotoxicity

Myocardial strain measured by echocardiography has been used to detect subclinical myocardial dysfunction in patients with breast cancer receiving chemotherapy [6-9]. Fallah-Rad et al. [7] reported that the global LV longitudinal and radial peak systolic strains decreased at 3 months, followed by a reduction in LVEF at 6 months in those who developed trastuzumab-related cardiotoxicity. Ho et al. [9] reported that the global LV longitudinal peak 
systolic strain was lower in asymptomatic breast cancer patients receiving anthracycline than in controls, even though the LVEF was broadly comparable between the two groups. Negishi et al. [6] proposed that changes in global LV longitudinal peak systolic strain could predict later reduction in LVEF in patients receiving trastuzumab, when taken into account alongside established clinical predictors. We found that CMR-based global LV peak systolic longitudinal and circumferential strains were slightly reduced at 3 months, followed by significant reductions at 6 months, suggesting that CMR-based myocardial strain studies may be effective means of detecting asymptomatic early trastuzumab-related cardiotoxicity. Specifically, the global LV longitudinal peak systolic strain, which closely reflects LV dysfunction [31], was the most reproducible parameter with a relatively low coefficient of variation, underlining its potential for the detection of subtle alterations in myocardial function attributable to trastuzumab. Changes in LV peak systolic strain were analogous to those in LVEF measured by CMR, but the predictive value of strain parameters for later LV dysfunction could not be determined. Our cohort included only asymptomatic patients whose LV function did not markedly decline during the observation period.

The RV free wall circumferential strain had also decreased by 6 months, but a firm conclusion about RV function cannot be drawn owing to the relatively low reproducibility of the test and the limited number of patients. The importance of RV function in predicting outcome in heart failure has been established [32]. Grover et al. [13] reported that RVEF decreased during anthracycline and/or trastuzumab therapy, and emphasized the importance of assessment of biventricular function in cardiotoxicity. Using our method, comprehensive evaluation of the RV parameters including myocardial strains may further illuminate the mechanisms of trastuzumab-related cardiotoxicity.

\section{Limitations of the study}

Our study has several limitations. First, the cohort was small, because only those consenting to participate in our study and not any other potentially conflicting study could be enrolled. However, the use of CMR-based strain studies in trastuzumab-related cardiomyopathy is novel and our findings are sufficient to suggest that CMR could have a role to play in routine clinical practice. Second, the strain parameters measured by CMR and echocardiography were not compared owing to the poor acoustic echocardiographic window in some patients; the relative merits of each technique are controversial and require further study [30]. Because the measurement of myocardial strains using echocardiography requires an adequate acoustic window, we did not compare strains measured by echocardiography with those measured by CMR. In our opinion, the limitations of echocardiography make a strong case for the use of CMR.

\section{Conclusions}

We describe the use of CMR-based biventricular myocardial strain studies for the serial evaluation of cardiac function in patients with early or locally advanced breast cancer receiving trastuzumab. Global LV peak systolic longitudinal and circumferential strains had significantly decreased by 6 months before recovering by 12 months; these changes were analogous to those in LVEF measured by CMR. RV parameters remained unchanged except for a marginal decrease in peak systolic circumferential strains.

Cardiac magnetic resonance imaging-based assessment of myocardial strain thus appears to be a useful means of detecting cardiotoxicity in patients receiving trastuzumab.

\section{Conflict of interest: None declared}

\section{References}

1. de Azambuja E, Procter MJ, van Veldhuisen DJ et al. Trastuzumab-associated cardiac events at 8 years of median follow-up in the herceptin adjuvant trial (big 1-01). J Clin Oncol, 2014; 32: 2159-2165.

2. Tocchetti CG, Ragone G, Coppola C et al. Detection, monitoring, and management of trastuzumab-induced left ventricular dysfunction: An actual challenge. Eur J Heart Failure, 2012; 14: 130-137.

3. Chavez-MacGregor M, Zhang N, Buchholz TA et al. Trastuzumab-related cardiotoxicity among older patients with breast cancer. J Clin Oncol, 2013; 31: 4222-4228.

4. Seidman A, Hudis C, Pierri MK et al. Cardiac dysfunction in the trastuzumab clinical trials experience. J Clin Oncol, 2002; 20: 1215-1221.

5. Otterstad JE, Froeland G, St John Sutton M, Holme I. Accuracy and reproducibility of biplane two-dimensional echocardiographic measurements of left ventricular dimensions and function. Eur Heart J, 1997; 18: 507-513.

6. Negishi K, Negishi T, Hare JL, Haluska BA, Plana JC, Marwick TH. Independent and incremental value of deformation indices for prediction of trastuzumab-induced cardiotoxicity. J Am Society Echocardiography, 2013; 26: 493-498.

7. Fallah-Rad N, Walker JR, Wassef A et al. The utility of cardiac biomarkers, tissue velocity and strain imaging, and cardiac magnetic resonance imaging in predicting early left ventricular dysfunction in patients with human epidermal growth factor receptor ii-positive breast cancer treated with adjuvant trastuzumab therapy. J Am Coll Cardiol, 2011; 57: 2263-2270. 
8. Hare JL, Brown JK, Leano R, Jenkins C, Woodward N, Marwick TH. Use of myocardial deformation imaging to detect preclinical myocardial dysfunction before conventional measures in patients undergoing breast cancer treatment with trastuzumab. Am Heart J, 2009; 158: 294-301.

9. Ho E, Brown A, Barrett P et al. Subclinical anthracycline- and trastuzumab-induced cardiotoxicity in the long-term follow-up of asymptomatic breast cancer survivors: A speckle tracking echocardiographic study. Heart (British Cardiac Society), 2010; 96: 701-707.

10. Oyama-Manabe N, Sato T et al. The strain-encoded (SENC) MR imaging for detection of global right ventricular dysfunction in pulmonary hypertension. Int J Cardiovasc Imaging, 2013; 29: 371-378.

11. Fallah-Rad N, Lytwyn M, Fang T, Kirkpatrick I, Jassal DS. Delayed contrast enhancement cardiac magnetic resonance imaging in trastuzumab induced cardiomyopathy. J Cardiovasc Magnetic Resonance, 2008; 10: 5.

12. Walker J, Bhullar N, Fallah-Rad N et al. Role of three-dimensional echocardiography in breast cancer: Comparison with two-dimensional echocardiography, multiple-gated acquisition scans, and cardiac magnetic resonance imaging. J Clin Oncol, 2010; 28: 3429-3436.

13. Grover S, Leong DP, Chakrabarty A et al. Left and right ventricular effects of anthracycline and trastuzumab chemotherapy: A prospective study using novel cardiac imaging and biochemical markers. Int J Cardiol, 2013; 168: 5465-5467.

14. Korosoglou G, Youssef AA, Bilchick KC et al. Real-time fast strain-encoded magnetic resonance imaging to evaluate regional myocardial function at 3.0 tesla: Comparison to conventional tagging. J Magnetic Resonance Imaging, 2008; 27: 1012-1018.

15. Teichholz LE, Kreulen T, Herman MV, Gorlin R. Problems in echocardiographic volume determinations: Echocardiographicangiographic correlations in the presence of absence of asynergy. Am J Cardiol, 1976; 37: 7-11.

16. Folland ED, Parisi AF, Moynihan PF, Jones DR, Feldman CL, Tow DE. Assessment of left ventricular ejection fraction and volumes by real-time, two-dimensional echocardiography. A comparison of cineangiographic and radionuclide techniques. Circulation, 1979; 60: 760-766.

17. Alfakih K, Plein S, Thiele H, Jones T, Ridgway JP, Sivananthan MU. Normal human left and right ventricular dimensions for mri as assessed by turbo gradient echo and steady-state free precession imaging sequences. J Magnetic Resonance Imaging, 2003; 17: 323-329.

18. Kramer CM, Barkhausen J, Flamm SD, Kim RJ, Nagel E. Standardized cardiovascular magnetic resonance imaging (CMR) protocols, society for cardiovascular magnetic resonance: Board of trustees task force on standardized protocols. J Cardiovasc Magnetic Resonance, 2008; 10: 35.

19. Osman NF, Sampath S, Atalar E, Prince JL. Imaging longitudinal cardiac strain on short-axis images using strain-encoded MRI. Magnetic Resonance Medicine, 2001; 46: 324-334.

20. Youssef A, Ibrahim el SH, Korosoglou G, Abraham MR, Weiss RG, Osman NF. Strain-encoding cardiovascular magnetic resonance for assessment of right-ventricular regional function. J Cardiovasc Magnetic Resonance, 2008; 10: 33.

21. Cerqueira MD, Weissman NJ, Dilsizian V et al., American Heart Association Writing Group on Myocardial S, Registration for Cardiac I. Standardized myocardial segmentation and nomenclature for tomographic imaging of the heart. A statement for healthcare professionals from the cardiac imaging committee of the council on clinical cardiology of the american heart association. Circulation, 2002; 105: 539-542.

22. McMurray JJ, Adamopoulos S, Anker SD et al. Guidelines ESCCfP. Esc guidelines for the diagnosis and treatment of acute and chronic heart failure 2012: The task force for the diagnosis and treatment of acute and chronic heart failure 2012 of the european society of cardiology. Developed in collaboration with the heart failure association (HFA) of the esc. Eur J Heart Failure, 2012; 14: 803-869.

23. Lawley C, Wainwright C, Segelov E, Lynch J, Beith J, McCrohon J. Pilot study evaluating the role of cardiac magnetic resonance imaging in monitoring adjuvant trastuzumab therapy for breast cancer. Asia-Pacific J Clin Oncol, 2012; 8: 95-100.

24. Friedrich MG, Sechtem U, Schulz-Menger J et al., International Consensus Group on Cardiovascular Magnetic Resonance in M. Cardiovascular magnetic resonance in myocarditis: A JAAC white paper. J Am Coll Cardiol, 2009; 53: 1475-1487.

25. Ewer MS, Lippman SM. Type ii chemotherapy-related cardiac dysfunction: Time to recognize a new entity. J Clin Oncol, 2005; 23: 2900-2902.

26. Serrano C, Cortes J, De Mattos-Arruda L et al. Trastuzumabrelated cardiotoxicity in the elderly: A role for cardiovascular risk factors. Ann Oncol, 2012; 23: 897-902.

27. Truong J, Yan AT, Cramarossa G, Chan KK. Chemotherapy-induced cardiotoxicity: Detection, prevention, and management. Canadian J Cardiol, 2014; 30: 869-878.

28. Strohm O, Schulz-Menger J, Pilz B, Osterziel KJ, Dietz R, Friedrich MG. Measurement of left ventricular dimensions and function in patients with dilated cardiomyopathy. J Magnetic Resonance Imaging, 2001; 13: 367-371.

29. Gardner BI, Bingham SE, Allen MR, Blatter DD, Anderson JL. Cardiac magnetic resonance versus transthoracic echocardiography for the assessment of cardiac volumes and regional function after myocardial infarction: An intrasubject comparison using simultaneous intrasubject recordings. Cardiovasc Ultrasound, 2009; 7: 38.

30. Bellenger NG, Burgess MI, Ray SG et al. Comparison of left ventricular ejection fraction and volumes in heart failure by echocardiography, radionuclide ventriculography and cardiovascular magnetic resonance; are they interchangeable? Eur Heart J, 2000; 21: 1387-1396.

31. Geyer H, Caracciolo G, Abe H et al. Assessment of myocardial mechanics using speckle tracking echocardiography: Fundamentals and clinical applications. J Am Soc Echocardiogr, 2010; 23: 351-369; quiz 453-355.

32. Ghio S, Gavazzi A, Campana $\mathrm{C}$ et al. Independent and additive prognostic value of right ventricular systolic function and pulmonary artery pressure in patients with chronic heart failure. J Am Coll Cardiol, 2001; 37: 183-188. 


\section{SUPPLEMENTARY MATERIALS}

\section{Detailed definition of data at baseline}

\section{Definition of data collection at baseline}

Hypertension was defined as current or previous treatment with anti-hypertensive medication. Diabetes mellitus was defined as current or previous treatment with antidiabetic medication (insulin or oral hypoglycemic drugs) or a hemoglobin A1c concentration of $\geq 6.5 \%$ [S1]. Dyslipidemia was defined as current or previous treatment with anti-dyslipidemic medication, a fasting serum low-density lipoprotein cholesterol concentration $\geq 160 \mathrm{mg} / \mathrm{dL}$, high-density lipoprotein cholesterol concentration $\leq 40 \mathrm{mg} / \mathrm{dL}$, or total cholesterol concentration $\geq 240 \mathrm{mg} / \mathrm{dL}$ [S2].

\section{Normal values of biomarkers}

Lower limit of detection levels and upper limit of normal values were $0.02-0.30 \mathrm{ng} / \mathrm{mL}$ for troponin I, $1.0-2.50 \mathrm{ng} / \mathrm{mL}$ for myosin light chain, and $4.0-18.40$ $\mathrm{pg} / \mathrm{mL}$ for B-type natriuretic peptide. Values below the lower limit of detection were treated as zero.

\section{References}

S1. International expert committee report on the role of the a1c assay in the diagnosis of diabetes. Diabetes Care, 2009; 32: 1327-1334.

S2. Expert Panel on Detection E, Treatment of High Blood Cholesterol in A. Executive summary of the third report of the national cholesterol education program (NCEP) expert panel on detection, evaluation, and treatment of high blood cholesterol in adults (adult treatment panel i ii). JAMA, 2001; 285: 2486-2497.

Supplementary Table S1. Detailed cardiac magnetic resonance protocol.

\begin{tabular}{lccccccc}
\hline Study & $\begin{array}{c}\text { Repetition } \\
\text { time }[\mathrm{ms}]\end{array}$ & $\begin{array}{c}\text { Echo time } \\
{[\mathrm{ms}]}\end{array}$ & $\begin{array}{c}\text { Flip angle } \\
{\left[{ }^{\circ}\right]}\end{array}$ & $\begin{array}{c}\text { Pixel area } \\
{[\mathrm{mm}]}\end{array}$ & $\begin{array}{c}\text { Voxel size } \\
{[\mathrm{mm}]}\end{array}$ & $\begin{array}{c}\text { Slice thickness } \\
{[\mathrm{mm}]}\end{array}$ & $\begin{array}{c}\text { Inter-slice gap } \\
{[\mathrm{mm}]}\end{array}$ \\
\hline Cine & 2.8 & 1.42 & 50 & $2.97 \times 1.69$ & $0.74 \times 0.74$ & 8 & 2 \\
T2 STIR & 2000 & 70 & 90 & $1.48 \times 1.97$ & $0.74 \times 0.74$ & 10 & - \\
LGE & 3.1 & 1.49 & 15 & $1.48 \times 1.90$ & $0.74 \times 0.74$ & 3 & - \\
SENC & 25 & 0.88 & 30 & $3.0 \times 3.0$ & $1.0 \times 1.0$ & 10 & - \\
\hline
\end{tabular}

*Reconstruction voxel size; STIR — short tau inversion recovery; LGE — late-gadolinium enhancement; SENC — strain-encoded imaging

Supplementary Table S2. Echocardiographic parameters at each time point.

\begin{tabular}{lccccccc}
\hline & Baseline & 3 months & $\mathbf{P}$ & $\mathbf{6}$ months & $\mathbf{P}$ & $\mathbf{1 2}$ months & $\mathbf{P}$ \\
\hline LV end-diastolic dimension [mm] & $43.2 \pm 2.9$ & $45.6 \pm 1.9$ & 0.069 & $45.3 \pm 3.0$ & 0.084 & $45.0 \pm 2.8$ & 0.088 \\
LV end-systolic dimension [mm] & $25.8 \pm 1.8$ & $28.1 \pm 3.4$ & 0.072 & $28.1 \pm 2.9$ & 0.072 & $28.5 \pm 2.2$ & $0.001^{*}$ \\
LV ejection fraction [\%] & $70.8 \pm 5.6$ & $68.7 \pm 7.1$ & 0.362 & $68.9 \pm 6.0$ & $0.037^{*}$ & $66.4 \pm 5.9$ & 0.113 \\
Intra-ventricular wall thickness [mm] & $9.5 \pm 1.1$ & $9.0 \pm 1.3$ & 0.252 & $9.4 \pm 0.7$ & 0.701 & $9.0 \pm 1.3$ & 0.369 \\
LV posterior wall thickness [mm] & $9.3 \pm 0.9$ & $9.4 \pm 1.3$ & 0.767 & $9.6 \pm 1.7$ & 0.270 & $8.9 \pm 1.3$ & 0.463 \\
\hline
\end{tabular}

Continuous variables are reported as the mean \pm standard deviation; ${ }^{*} \mathrm{P}<0.05$ vs. baseline; $L V-$ left ventricle 
Supplementary Table S3. Cardiac magnetic resonance parameters at each time point.

\begin{tabular}{|c|c|c|c|c|c|c|c|}
\hline & Baseline & 3 months & $\mathbf{P}$ & 6 months & $\mathbf{P}$ & 12 months & $\mathbf{P}$ \\
\hline \multicolumn{8}{|l|}{ Conventional parameters } \\
\hline LV end-diastolic volume index $\left[\mathrm{mL} / \mathrm{m}^{2}\right]$ & $56.8 \pm 7.5$ & $59.7 \pm 7.0$ & 0.114 & $58.4 \pm 3.7$ & 0.424 & $59.0 \pm 7.8$ & 0.425 \\
\hline LV end-systolic volume index $\left[\mathrm{mL} / \mathrm{m}^{2}\right]$ & $24.8 \pm 6.7$ & $28.4 \pm 7.2$ & $0.024^{*}$ & $30.7 \pm 5.4$ & $0.004^{*}$ & $30.5 \pm 5.7$ & $0.002 *$ \\
\hline LV ejection fraction [\%] & $68.4 \pm 6.6$ & $65.4 \pm 8.7$ & 0.176 & $61.7 \pm 8.7$ & $0.004^{*}$ & $62.9 \pm 7.8$ & $0.017^{*}$ \\
\hline LV wall mass index $\left[\mathrm{g} / \mathrm{m}^{2}\right]$ & $23.4 \pm 4.7$ & $22.9 \pm 4.3$ & 0.761 & $25.2 \pm 4.3$ & 0.343 & $23.2 \pm 5.1$ & 0.934 \\
\hline RV end-diastolic volume index $\left[\mathrm{mL} / \mathrm{m}^{2}\right]$ & $68.7 \pm 16.3$ & $72.4 \pm 15.0$ & 0.256 & $72.0 \pm 12.3$ & 0.443 & $73.0 \pm 14.9$ & 0.204 \\
\hline RV end-systolic volume index $\left[\mathrm{mL} / \mathrm{m}^{2}\right]$ & $46.0 \pm 13.8$ & $46.8 \pm 13.2$ & 0.780 & $47.2 \pm 11.8$ & 0.703 & $46.5 \pm 11.6$ & 0.876 \\
\hline RV ejection fraction [\%] & $30.7 \pm 9.0$ & $31.3 \pm 8.5$ & 0.888 & $31.4 \pm 7.3$ & 0.880 & $31.0 \pm 7.4$ & 0.814 \\
\hline \multicolumn{8}{|l|}{ Peak systolic strain } \\
\hline Global LV, longitudinal [\%] & $-21.1 \pm 1.7$ & $-20.1 \pm 1.5$ & 0.128 & $-19.5 \pm 1.0$ & $0.039 *$ & $-20.7 \pm 1.2$ & 0.622 \\
\hline Global LV, circumferential [\%] & $-23.4 \pm 1.8$ & $-21.9 \pm 2.7$ & 0.124 & $-21.6 \pm 2.5$ & $0.036^{*}$ & $-21.8 \pm 2.3$ & 0.080 \\
\hline RV free wall, longitudinal [\%] & $-23.3 \pm 1.3$ & $-22.6 \pm 1.2$ & 0.168 & $-22.3 \pm 1.5$ & 0.096 & $-22.7 \pm 1.7$ & 0.384 \\
\hline RV free wall, circumferential [\%] & $-20.9 \pm 2.4$ & $-21.2 \pm 2.4$ & 0.749 & $-19.1 \pm 2.3$ & $0.049 *$ & $-20.0 \pm 1.6$ & 0.276 \\
\hline
\end{tabular}

Continuous variables are reported as the mean \pm standard deviation; ${ }^{*} P<0.05$ vs. baseline; LV — left ventricle; RV - right ventricle

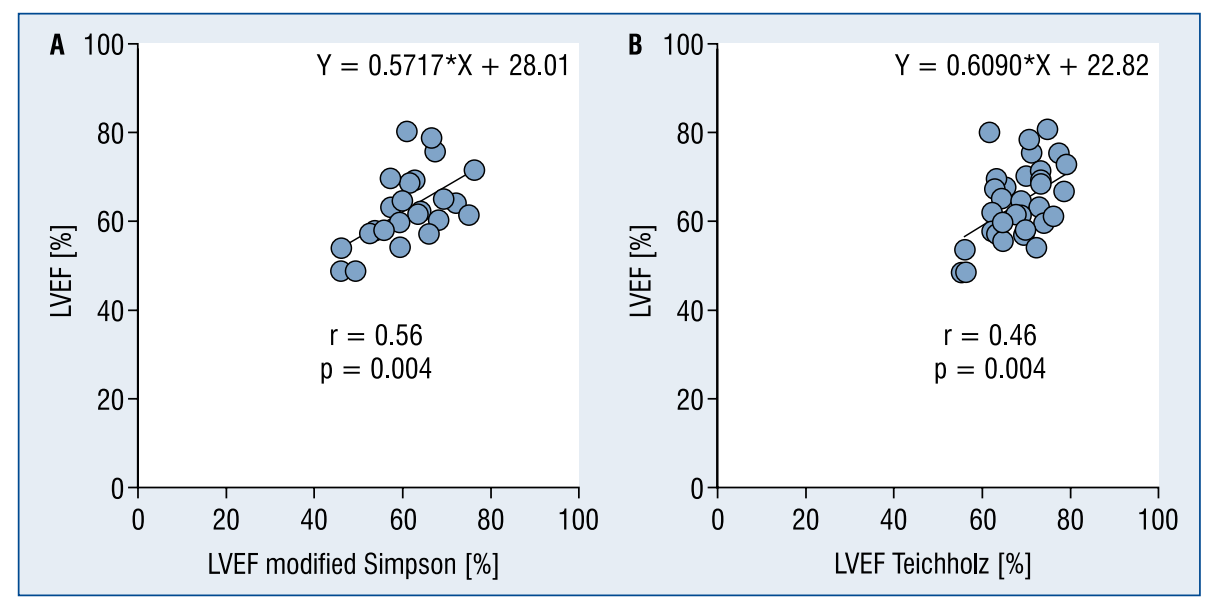

Supplementary Figure S1. Linear regression plots comparing left-ventricular ejection fraction (LVEF) measured by cardiac magnetic resonance (CMR) and echocardiography; A. Modified Simpson method; B. Teichholz method; Pearson's correlation analysis. 